\title{
Von den gemeinsamen Wurzeln meines ärztlichen und künstlerischen Schaffens
}

\author{
W. Furrer
}

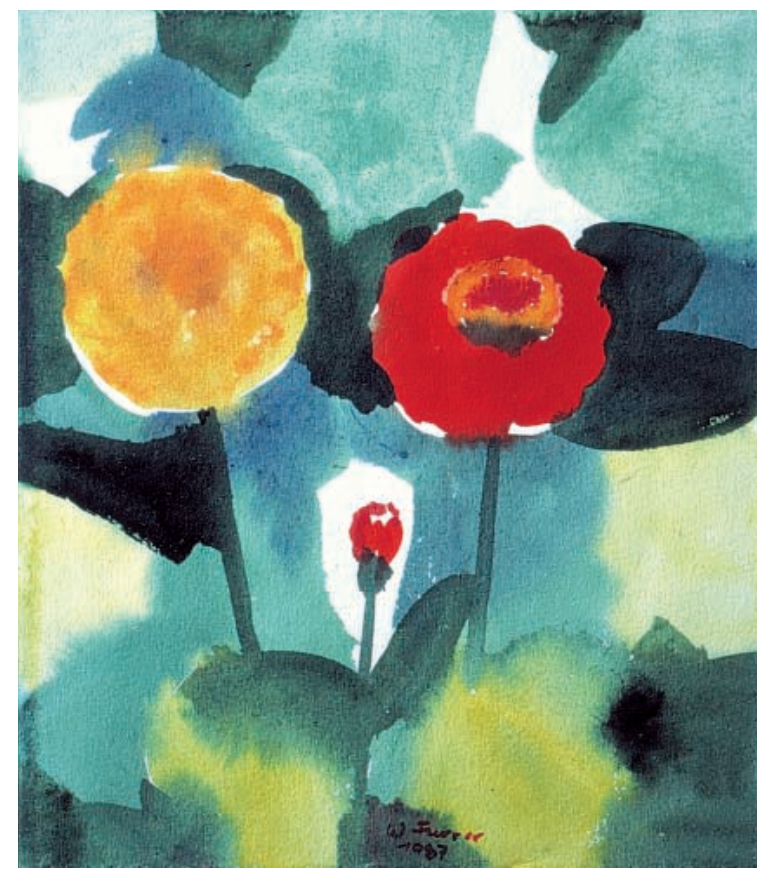

Wie vielschichtig in der psychotherapeutischen Praxis das Kommunikationsgeschehen sein kann, will ich an einigen Bilddokumenten aufzeigen, welche die unbewussten Vorgänge in der Arzt-Patient-Beziehung sichtbar machen. Es hat sich in besonderen Therapiesituationen ergeben, dass sowohl der Analysand als auch der Arzt beide vor sich hinkritzelten. Bei dieser therapeutischen Massnahme ging es nicht um thematische Darstellung, sondern um eine psychische Auflockerung bei solchen depressiven Patienten, die sich verbal nicht mehr artikulieren konnten. Die graphischen Ergebnisse waren verblüffend: Die Kritzeleien von Arzt und Patient wiesen nämlich eindrückliche Ähnlichkeiten auf, obwohl der Einblick auf das Blatt des anderen während des Kritzelns nicht hatte stattfinden können. Die nonverbalen Korrespondenzen (Abbildungen aus [1, 2]) sind das Ergebnis einer unbewussten Kommunikation zwischen Arzt und Patient.

Korrespondenz:

Dr. med. Walter Furrer

Auf Weinbergli 15

CH-6005 Luzern

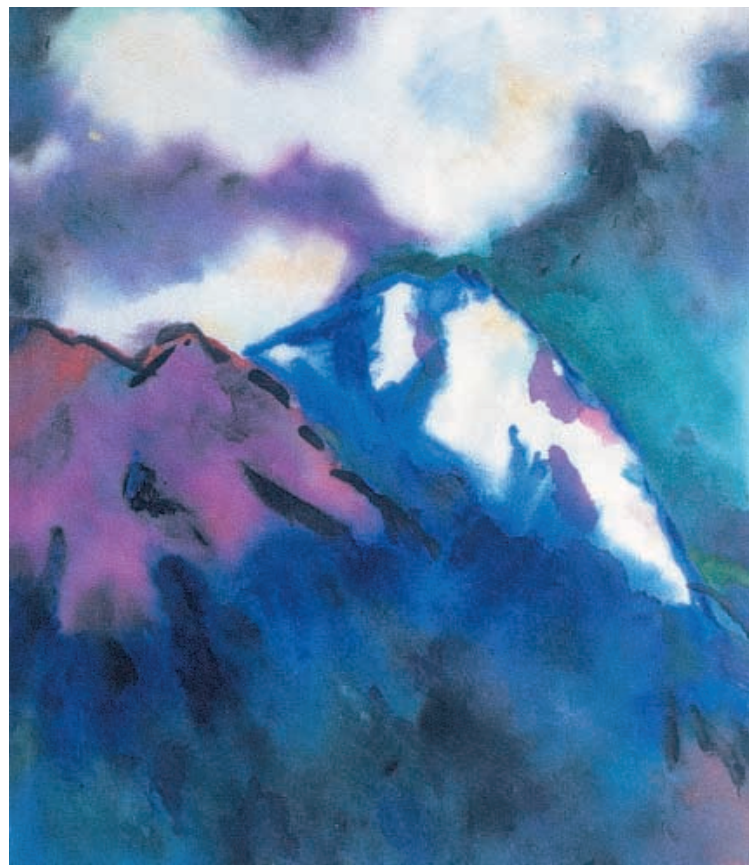

Diese wissenschaftlich dokumentierte unbewusste Einfühlung in das Gegenüber ist eine ganzheitlichintuitive Fähigkeit, die sowohl beim Psychotherapeuten als auch beim Künstler vorhanden sein muss.

Für das Gelingen der Psychotherapie bedarf es einer Grundvoraussetzung: sich immer wieder von neuem öffnen für alles, was dem Arzt vom Patienten her begegnet. So muss man in dieser dialogischen Situation sogar seine wohlfundierten Theorien und Behandlungstechniken vergessen können, um wirklich unvoreingenommen wahrzunehmen.

Die Bereitschaft, die Wirklichkeit sein zu lassen und sich zeigen zu lassen, setzt eine seelische Empfänglichkeit voraus, die bei der heute üblichen Geisteshaltung des Zugreifens und Beherrschenwollens fast verloren gegangen ist. Auf anschauliche Weise bringt der Traum einer Analysandin dies zum Ausdruck: «Dr. Furrer verfügt über einen Röntgenapparat, mit dem man die innere Struktur der Person entdecken kann, und nach diesen Strukturen malt er auch seine Bilder."

Das zentrale Thema meines Lebens ist die menschliche Beziehung. Um dieses Thema kreist seit Jahrzehnten meine wissenschaftliche Forschung; in der täglichen Arbeit als Psychotherapeut ist Kommuni- 
kation das eigentlich Tragende der ärztlichen Kunst, und auch im künstlerischen Schaffen liegen mir die Themen der Beziehung besonders am Herzen: Arzt-Patient, Mutter-Kind, Gespräch, Freundschaft, Fürsorge u.ä. Auch in meinen Landschaften und Blumenaquarellen ist das Thema enthalten.

Auf der Grundlage dieser Offenheit kann sich dann in einem kreativen Umformungsprozess die Erkenntnis des Wesentlichen vollziehen, die den Kern der Sache fassbar macht. Analog gestaltet sich der schöpferische Vorgang auch in der Malerei: in beiden Bereichen ist es das Schwierigste, sich immer wieder neu bereitzumachen und offenzuhalten für das je Einmalige und Substantielle dessen, was sich begibt. Dann aber kann es geschehen, dass mir die Bilder von selbst kommen, spontan aufsteigend aus den inneren Quellen des Unbewussten.

Es bleibt dann noch die anders geartete Aufgabe der kreativen Umsetzung ins konkrete Werk. Erreichen kann man eine solche Offenheit und Empfänglichkeit ein Stück weit durch die Ausweitung des Erfahrungs- und Erlebnisfeldes bis in die Tiefenbereiche des Unbewussten, wie sie sich durch die Lehranalyse und das tägliche Studium der Träume und der übrigen Manifestationen des Unbewussten ergibt; aber darüber hinaus ist es notwendig, die gesamte Lebensgestaltung auf das Ganzheitliche und auf das Wesen der Dinge auszurichten. Sowohl der Arzt, der seinen Dienst am leidenden Menschen nach besten Möglichkeiten erfüllen will, als auch der Künstler, der in seinen schöpferischen Werken neue Dimensionen

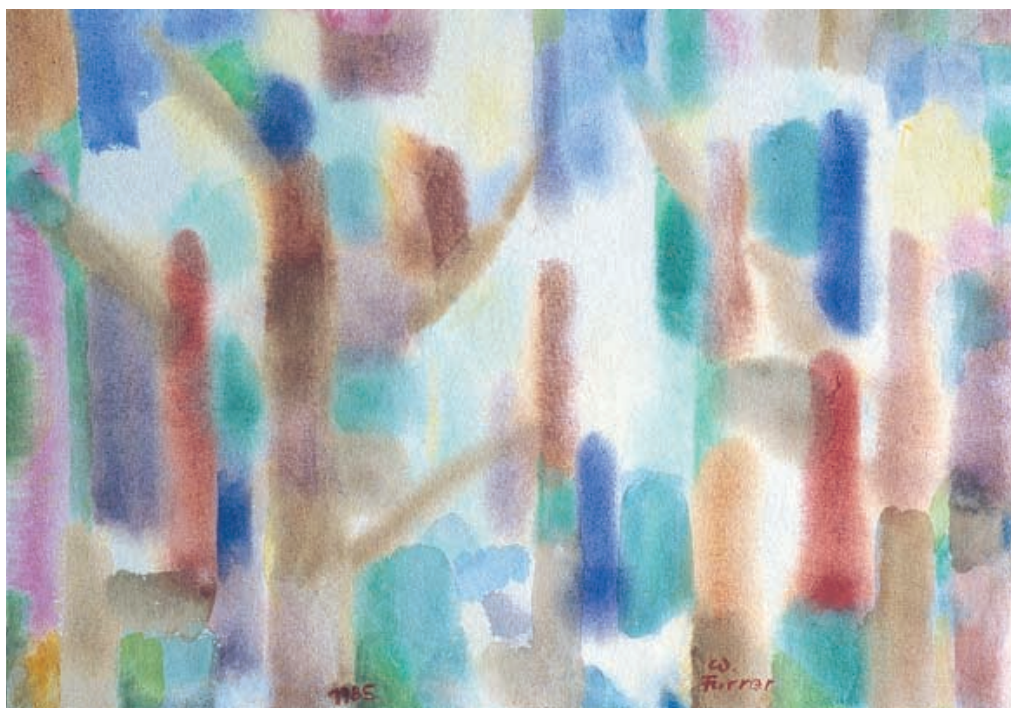

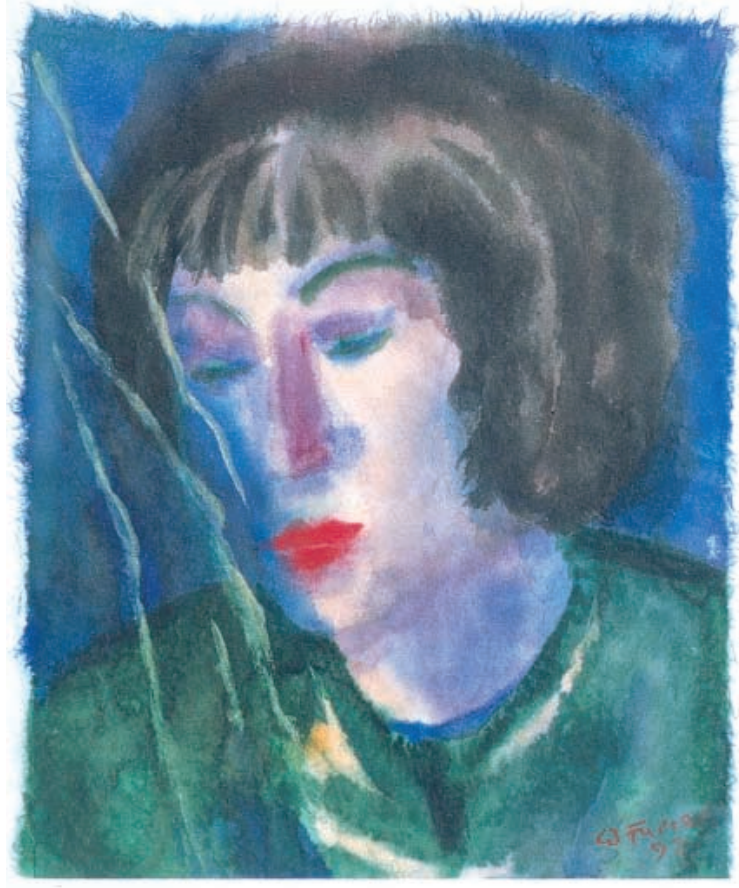

erschliesst und eine geistige Botschaft an die Welt weiterzugeben hat, stellt das Ganze seines Lebens in den Dienst der Sache. Unter diesen Voraussetzungen kann man die Fähigkeit entwickeln, sich vom Unbewussten her inspirieren und leiten $\mathrm{zu}$ lassen, was gar nicht einfach ist, weil es einen teilweisen Verzicht auf die "sicheren" Werte der Logik, des Wissens und der rationalen Erkenntnis verlangt, welche in der modernen wissenschaftlichen Welt als das höchste gelten.

\section{Literatur}

1 Furrer W. Neue Wege zum Unbewussten. Bern: Huber; 1970.

2 Furrer W. Unbewusste Kommunikation. Bern: Huber; 1977.

"Landschaften von Nordstrand bis Marokko" von Walter Furrer. Galerie Weinberg, Auf Weinbergli 15, Luzern. 13. Oktober bis Weihnachten 2001. Jeden Samstag, 14.30-18.00 Uhr, sonst auf Anfrage (Tel. 04136022 77).

Bilder im Internet: http:// furrer.ch.to 